\title{
A STUDY OF INCLUSTVE PROTON POLARIZATION
}

\section{Experimenters}

M. Corcoran, S. C. Ems, F. Fredericksen, S. W. Gray,

H. A. Neal, H. O. Ogren, D. R. Rust, and J. R. Sauer

\author{
Department of Physics \\ Indiana University \\ Bloomington, Indiana 47401
}

\section{Spokesman: H. Ogren}

\section{Abstract}

We propose to measure the polarization of the outgoing proton In the inclusive reaction $p+p+p^{4}+x$ in the region $.98>x_{R}>.6$. The measurements would include beam momenta from $\mathrm{P}_{\mathrm{LAB}}=50 \mathrm{GeV} / \mathrm{c}$ to $\mathrm{P}_{\mathrm{LAB}}=400 \mathrm{GeV} / \mathrm{c}$ at $t=-.3(\mathrm{GeV} / \mathrm{c})^{2},-.8(\mathrm{GeV} / \mathrm{c})^{2},-1.4(\mathrm{GeV} / \mathrm{c})^{2}$, and $-3.0(\mathrm{GeV} / \mathrm{c})^{2}$ over the range $.6<x_{\mathrm{F}}<.95$. The experiment would use the existing E313 polarimeter and internal target spectrometer. 


\section{Physics Interest}

The extension of proton polarization measurements from pp elastic to inclusive processes is a very natural one. The present E313 measurement studies the process shown in Figure (1a) as a function of $s$ and $t$. The measurements we propose extend this to include inclusive processes shown In Figure (1b) where the polarization can be studied as a function of $s$, $t, M^{2}$ (triple regge formalism) or $x, p_{T^{*}}$

In the kinematic region $s+\infty, M^{2} \rightarrow \infty, s / M^{2}+\infty$, and $s>t(.95>x>.8)$ the inclusive cross section for Figure (1b) can be related to the triple regge amplitude in the Regge-Mueller formalism. The triple regge amplitude is given in Figure $(1 c)$. In this $x$ region the polarization measurements can provide a direct test of several triple Regge predictions.

The polarization of the inclusive proton results from the interference of a flip and nonflip exchanged trajectory and, as such, in this picture would measure the PRR contribution to the triple regge amplitude. Terms of this type are expected ${ }^{(1,2,3)}$ to be a function of $1 / M^{2} \sqrt{s}$, where $M$ is the mass of the state recolling against the proton.

Outside the triple regge region $(x<.8)$ we know of no model that makes predictions of proton polarlzations. However, recent results from the hyperon beam at FNAL ${ }^{(4)}$ Indicate an Inclusive $\Lambda^{\circ}$ polarization greater than $28 \%$ in the region $\mathrm{p}_{\mathrm{T}} \rightarrow 1-1.5 \mathrm{GeV} / \mathrm{c}, \mathrm{x} \sim .7 \rightarrow .4$. These large effects are totally unexpected, Indtcating, perhaps, the predominance of a completely new process at high $\mathrm{p}_{\mathrm{T}}$. A systematic study of the polarization of protons in this region of phase space is therefore very important. 


\section{Data Collection}

a) Kinematics

We propose to measure the inclusive proton polarization at four $t$ values, $t=0.3,-0.8,-1.4$, and $-3.0(\mathrm{GeV} / \mathrm{c})^{2}$ as a function of recoll mass, $M$, for three beam energies between 50 and $400 \mathrm{GeV}$. The corresponding $x_{R}, P_{T}$ values are indicated in Table $I$.

The existing internal target superconducting spectrometer and E313 polarimeter (Figure 2) would be used for this measurement. The polarimeter with a $\pm 5 \%$ momentum acceptance determines the proton polarization from the asymetric "double scattering" of the proton by a carbon block. The spectrometer is remotely moveable piecewise from $\theta=36^{\circ}$ to $\theta=90^{\circ}$.

The kinematics ( $\theta$ vs. $x$ ) for fixed $t$, (i.e., fixed proton total recoil momentum) is shown in Figure 3. For the triple regge region $(x>.8)$ the angular range is restricted to $72^{\circ}>\theta>50^{\circ}$.

At fixed $t$ and $s$ each $\theta$ setting corresponds to a missing mass indicated in Figure 4. For $\mathrm{p}_{\mathrm{BEAM}}=400 \mathrm{GeV} / \mathrm{c}, \mathrm{t}=-.3$, the angular range $70^{\circ}<\theta<54^{\circ}$ spans missing masses up to $\mathrm{M}_{\mathrm{X}}^{2}=120(\mathrm{GeV} / \mathrm{c})^{2}$. The corresponding mass acceptance (FWHM) is shown in Figure 5.

b) Using the warm hydrogen fet at $\mathrm{CO}$, data can be collected at $\mathrm{E}_{1 \mathrm{ab}}=50$, 150,300 , and $400 \mathrm{GeV}$ during one machine ramp. Four separate magnet settings are required (corresponding to $t=-.3,-.8,-1.5,-3.0$ ) and 9 separate spectrometer angles. The $\mathrm{p}_{\mathrm{T}}$ and $\mathrm{x}$ distributions for the proposed data collection points are shown in Figure 6 .

c) Rates

We have calculated the proton inclusive ylelds based on an extensive fit to proton inclusive invarlant cross sections by Taylor et al. (3) The 
yields are based on the present 313-198 configuration assuming a beam-jet luminosity of $10^{34} \mathrm{~cm}^{-2}$ per jet pulse.

Using the hardware processor utilized In E313 and the reconstruction efficlency obtained from elastic events, the data collection times for a $2 \%$ measurement of polarization can be calculated and are indicated in Table I.

The existing TOF measurement separates particles unambiguously at all data collection points.

\section{Requirements}

The existing E313 polarimeter and internal target superconducting spectrometer can be used for this experiment without modification. E313 has proven its capability to measure polarizations smaller than $1 \%$ and is wellsuited for this measurement.

We expect to be able to begin this experiment when E313 has completed a first scan of the $s$ dependence of elastic recoll protons at several $t$ values in the spring, 1977. Alternatively, we could run interspersed with E313.

Beam time requirements are 840 hours to complete the entire data scan with a $2 \%$ polarization measurement. 


\section{REFERENCES}

1. R. D. Field and G. C. Fox, Nucl. Phys. B80, 367 (1974).

2. R. D. Field, ANL Summer Studies on High-Energy Physics with Polarized Beams, 1974, VI.

3. F. Taylor, et al., Fermilab Publication 75-90-EXP.

4. F. Paige and D. Sidhu, BNL 21695.

5. G. Bunce, et a1., PRL 36, 1113 (1976). 


\begin{tabular}{|c|c|c|c|c|c|c|c|c|c|c|c|c|c|c|c|c|}
\hline \multirow{2}{*}{$\begin{array}{l}t \\
\theta\end{array}$} & \multicolumn{4}{|c|}{$-.3(\mathrm{GeV} / \mathrm{c})^{2}$} & \multicolumn{4}{|c|}{$-.8(\mathrm{GeV} / \mathrm{c})^{2}$} & \multicolumn{4}{|c|}{$-1.5(\mathrm{GeV} / \mathrm{c})^{2}$} & \multicolumn{4}{|c|}{$-3.0(\mathrm{GeV} / \mathrm{c})^{2}$} \\
\hline & $\begin{array}{c}\mathrm{M} \\
\mathrm{GeV} / \mathrm{c}^{2}\end{array}$ & $\mathbf{x}_{\mathbf{R}}$ & $\begin{array}{c}\mathbf{p}_{\mathbf{T}} \\
\mathrm{GeV} / \mathrm{c}\end{array}$ & $\begin{array}{l}\mathrm{T}(2 \%) \\
\text { hours }\end{array}$ & $\begin{array}{c}\mathrm{M} \\
\mathrm{GeV} / \mathrm{c}^{2}\end{array}$ & $\mathbf{x}_{\mathrm{R}}$ & $\begin{array}{c}\mathrm{p}_{\mathrm{T}} \\
\mathrm{GeV} / \mathrm{c}\end{array}$ & $\begin{array}{l}\mathrm{T}(2 \%) \\
\text { hours }\end{array}$ & $\begin{array}{c}\mathrm{M} \\
\mathrm{GeV} / \mathrm{c}^{2}\end{array}$ & $\mathbf{x}_{\mathbf{R}}$ & $\begin{array}{c}\mathrm{p}_{\mathrm{T}} \\
\mathrm{GeV} / \mathrm{c}\end{array}$ & $\begin{array}{r}\mathrm{T}(2 \%) \\
\vdots \\
\text { hours }\end{array}$ & $\begin{array}{c}M \\
\mathrm{GeV} / \mathrm{c}^{2}\end{array}$ & ${ }^{x_{R}}$ & $\begin{array}{c}\mathrm{p}_{\mathrm{T}} \\
\mathrm{GeV} / \mathrm{c}\end{array}$ & $\begin{array}{l}\mathrm{T}(2 \%) \\
\text { hours }\end{array}$ \\
\hline $66^{\circ}$ & 7.6 & .93 & .55 & 31 & & & & & & & & & & & & \\
\hline $62^{\circ}$ & & & & & 5.2 & .96 & .88 & 39 & & & & & & & & \\
\hline $58^{\circ}$ & 10.7 & .88 & .51 & 31 & 9.0 & .90 & .85 & 37 & & & & & & & & \\
\hline $54^{\circ}$ & & & & & 11.1 & .83 & .81 & 31 & 8.1 & .94 & 1.17 & 111 & & & & \\
\hline $50^{\circ}$ & 13.0 & .78 & .46 & 31 & & & & & 10.5 & .86 & 1.12 & 63 & & & & \\
\hline $46^{\circ}$ & & & & & 14.5 & .72 & .72 & 31 & 13.0 & .78 & 1.07 & 31 & & & & \\
\hline $42^{\circ}$ & & & & & & & & & 15.1 & .70 & 1.00 & 31 & 11.0 & .85 & 1.60 & $80(4 \%)$ \\
\hline $38^{\circ}$ & & & & & & & & & & & & & 14.4 & .73 & 1.47 & 119 \\
\hline $36^{\circ}$ & 15.7 & .67 & .35 & 31 & 17.4 & .60 & .59 & 31 & 17.4 & .60 & .86 & 31 & $15.7^{\prime}$ & .68 & 1.40 & 77 \\
\hline Tota & & & $124 \mathrm{~h}$ & lours & & & & 169 & & & & 267 & & & 27 & \\
\hline
\end{tabular}

TOTAL 836 hours

T A B L E I 

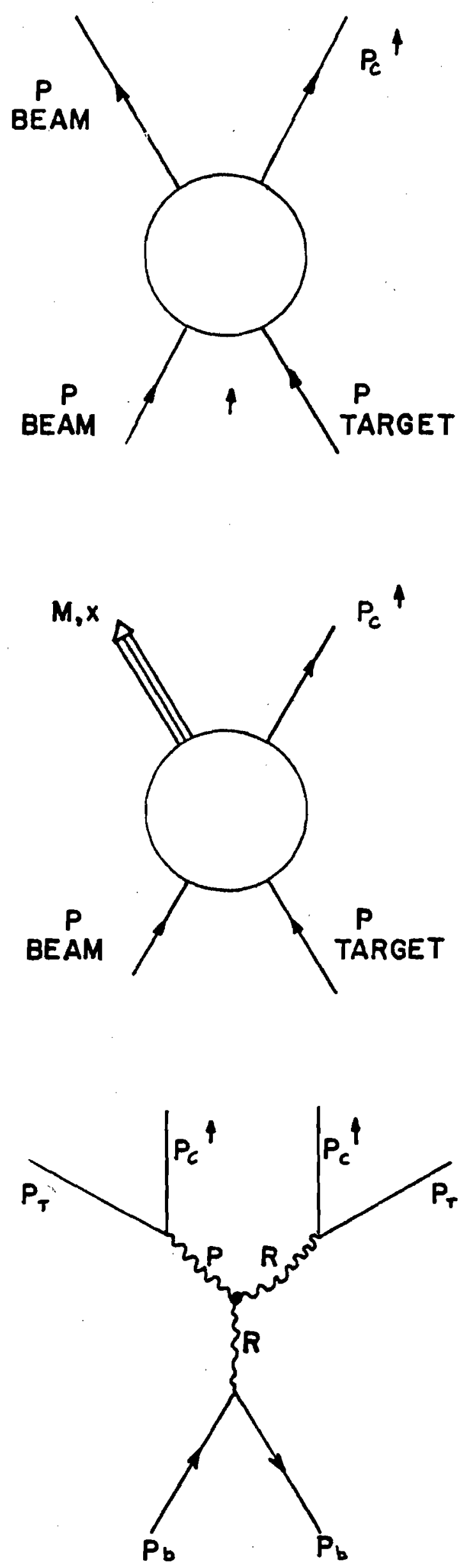

FIGURE 1 


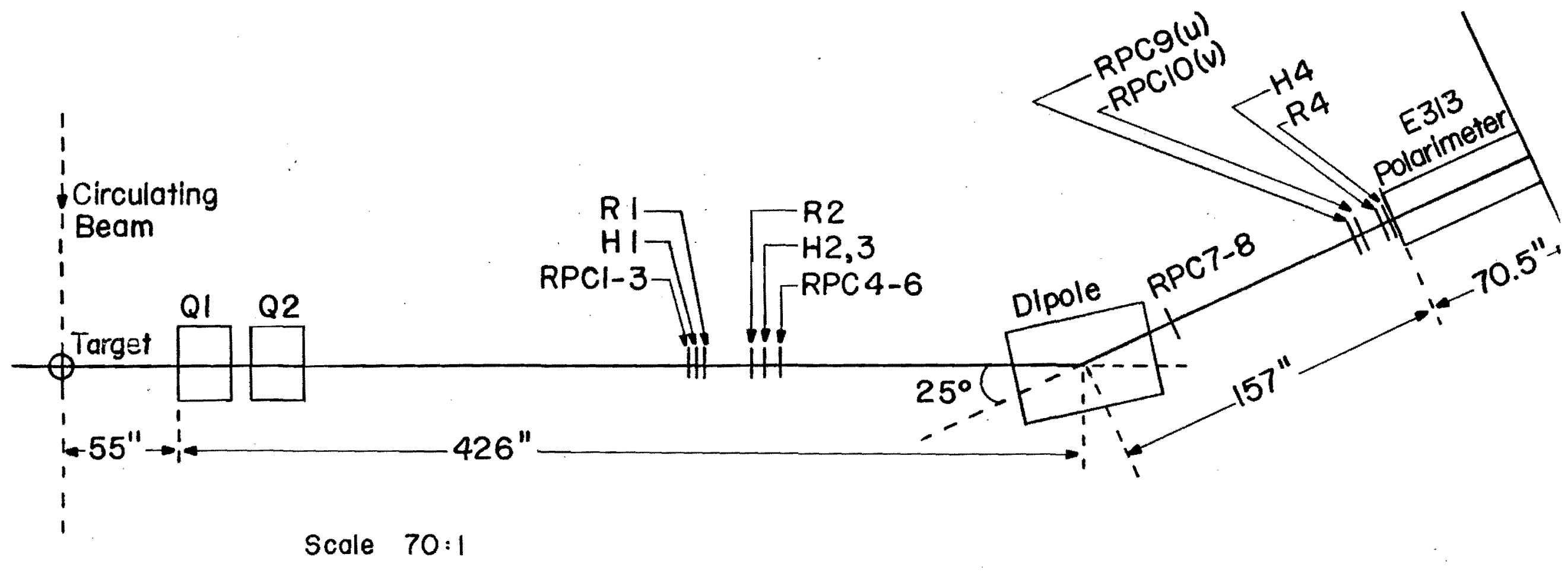
SPECTROMETER 

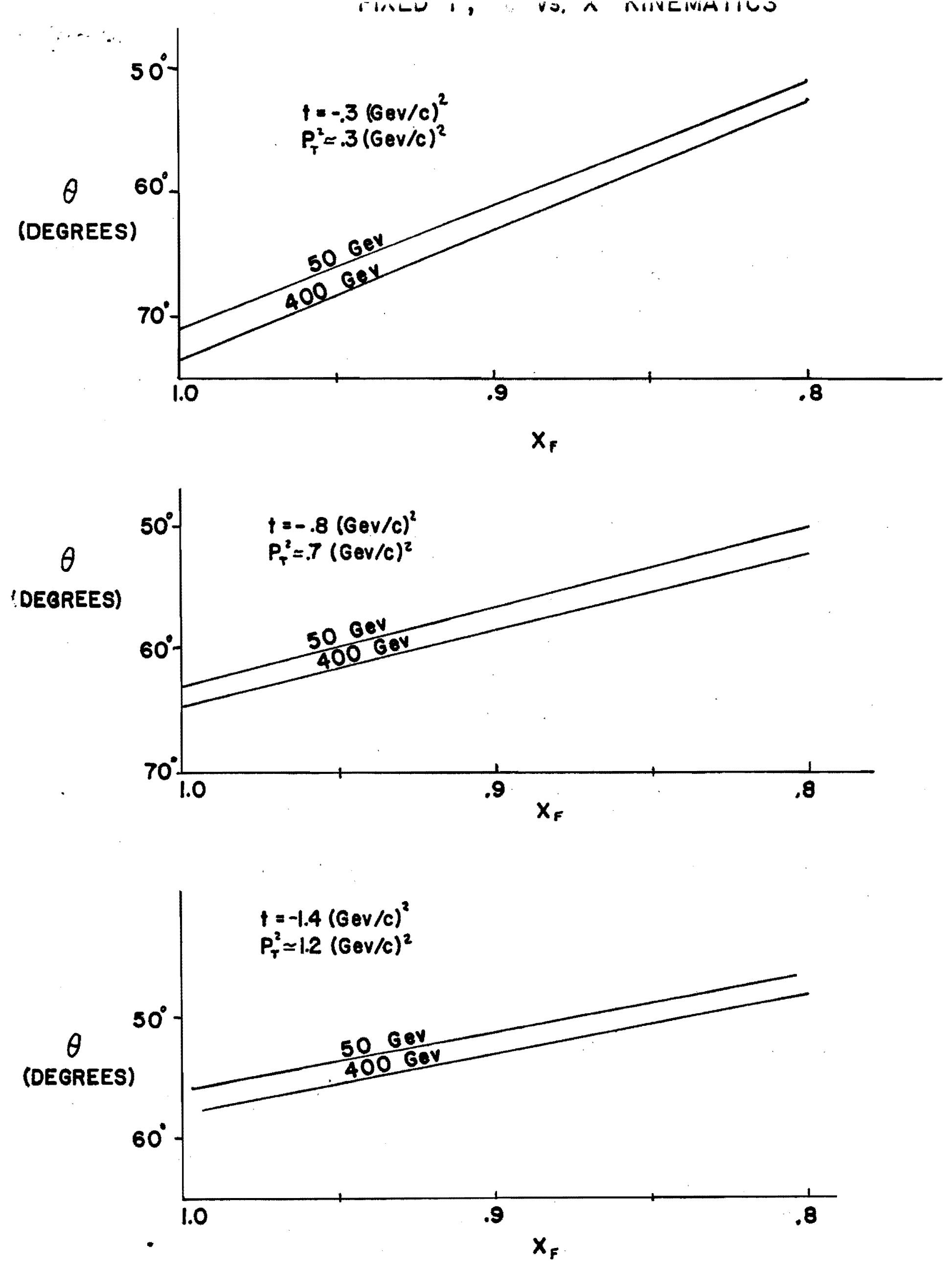

FIGURE 3 


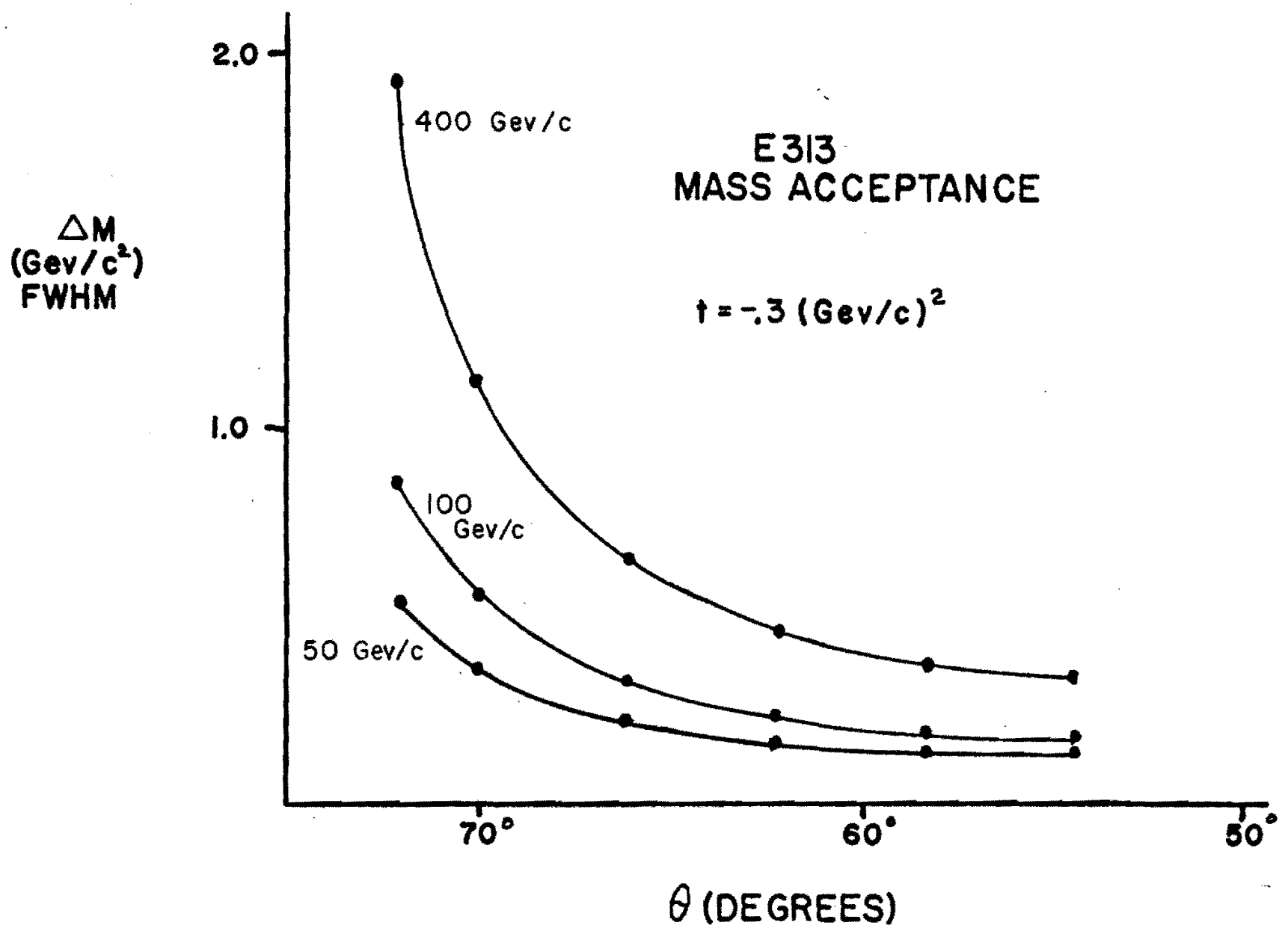

FIGURE 5 


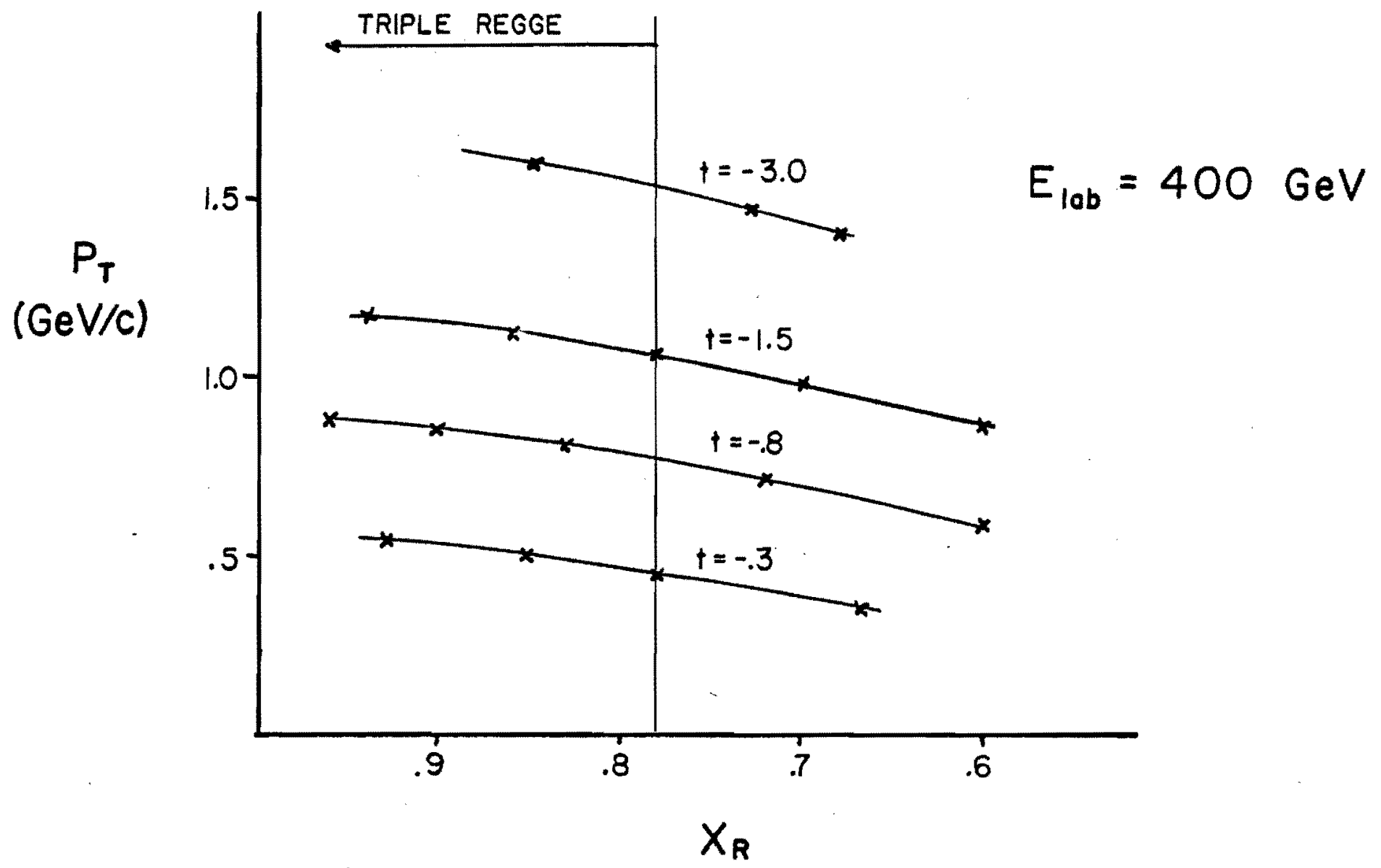

FIGURE 6 